\title{
Topological Scenario for Stripe Formation in Manganese Oxides
}

\author{
Takashi Hotta ${ }^{1,2}$, Yasutami Takada ${ }^{2}$, Hiroyasu Koizumi ${ }^{3}$, and Elbio Dagotto ${ }^{1}$ \\ 1 National High Magnetic Field Laboratory, Florida State University, Tallahassee, FL 32306 \\ 2 Institute for Solid State Physics, University of Tokyo, 7-22-1 Roppongi, Minato-ku, Tokyo 106-8666, Japan \\ ${ }^{3}$ Faculty of Science, Himeji Institute of Technology, Kamigori, Ako-gun, Hyogo 678-1297, Japan
}

(August 13, 1999)

\begin{abstract}
The spin-charge-orbital complex structures of manganites are studied using topological concepts. The key quantity is the "winding number" $w$ associated with the Berry-phase connection of an $e_{g}$ electron parallel-transported through Jahn-Teller centers, along zigzag one-dimensional paths in an antiferromagnetic environment of $t_{2 g}$ spins. From these concepts, it is shown that the "bi-stripe" and "Wigner-crystal" states observed experimentally have different $w$ 's. Predictions for the spin structure of the charge-ordered states for heavily doped manganites are discussed.
\end{abstract}

71.70.Ej,71.15.-m,71.38.+i,71.45.Lr

The curious static patterns in the spin, charge, and orbital densities observed in manganites are currently attracting much attention [1]. In $\mathrm{La}_{1-x} \mathrm{Ca}_{x} \mathrm{MnO}_{3}$, the CE-type antiferromagnetism (AFM) has been observed at $x=1 / 2$ since the 1950 's [2], but recently a similar structure has been proposed at $x=2 / 3$ based on neutron diffraction experiments [3]. In these AFM structures, the $t_{2 g}$ spins align in parallel along zigzag-shaped onedimensional (1D) paths in the $a$-axis direction, while they are antiparallel across these paths, which are stacked in the $b$-axis direction. This spin arrangement here is called the "stripe-AFM" (S-AFM) structure.

Interestingly enough, the charge and orbital ordering (COO) observed experimentally is always concomitant with this S-AFM phase. At $x=1 / 2$, the COO has been confirmed by the synchrotron X-ray diffraction experiment 顿. At $x=2 / 3$, however, two different COO patterns have been reported; the "bi-stripe" (BS) structure [5], in which the main building block of the COO pattern at $x=1 / 2$ persists even at $x=2 / 3$, and the "Wignercrystal" (WC) structure [3], in which the COO occurs with the distance between charges maximized. The appearance of the two different structures indicates that (1) the corresponding energies are very close to each other, namely, the ground state has (quasi-)degeneracy and that (2) the conversion between them is prohibited by a large energy barrier. Under these circumstances, it is of limited relevance the determination of which of the two states is better energetically based on some model Hamiltonian.

In such a subtle situation, we focus on the origin of the near BS-WC degeneracy, rather than calculating which one is the true ground state. We follow the strategy of labeling these (quasi-)degenerate ground states in terms of a physically-motivated quantity which does not necessarily manifest itself in the Hamiltonian $H$. Specifically we focus our attention on the important role of the $1 \mathrm{D}$ conducting zigzag paths in the $a-b$ basal plane, and considered parallel-transport of an $e_{g}$ electron along these paths through the Jahn-Teller (JT) centers composed of
$\mathrm{MnO}_{6}$ octahedra. The transport invokes the Berry-phase connection and we can introduce the "winding number" $w$ as a direct consequence of topological invariance which should be conserved irrespective of the details of $H$.

In this Letter, we propose that such a topological invariance is a key concept to understand the complex states of manganites since we observe that $w$ is always a good index to label the (quasi-)degenerate S-AFM states for $x \geq 1 / 2$. In fact, it is found that if the energies for various paths considered here are plotted, its distribution contains a multifold-band structure indexed by $w$. The observed WC and BS structures belong to two topologically different classes, characterized by the 1D paths with $w=1$ and $w=x /(1-x)$, respectively. The conversion between the $\mathrm{WC}$ and $\mathrm{BS}$ states is hindered by a large energy barrier due to this topological difference.

Consider $e_{g}$ electrons coupled to both localized $t_{2 g}$ spins, with the Hund's rule coupling $J_{\mathrm{H}}$, and JT distortions of the $\mathrm{MnO}_{6}$ octahedra. Since it is the largest characteristic energy among those considered here, $J_{\mathrm{H}}$ is taken to be infinite for simplicity. This implies that the spin of each $e_{g}$ electron at a Mn site aligns completely in parallel with the direction of the $t_{2 g}$ spins at the same site. Thus, the spin degrees of freedom are effectively lost for the $e_{g}$ electrons, and the spin index will be dropped hereafter. Since experimentally it is known that the $t_{2 g}$ spins are antiparallel along the $c$-axis, we can assume that the $e_{g}$ electrons can move only in the $a-b$ plane.

The above situation is well described in terms of $H$ as

$$
\begin{aligned}
H & =-\sum_{\mathbf{i} \mathbf{a} \gamma \gamma^{\prime}} t_{\gamma \gamma^{\prime}}^{\mathbf{a}} d_{\gamma \mathbf{i}}^{\dagger} d_{\gamma^{\prime} \mathbf{i}+\mathbf{a}}+J^{\prime} \sum_{\langle\mathbf{i}, \mathbf{j}\rangle} \mathbf{S}_{\mathbf{i}} \cdot \mathbf{S}_{\mathbf{j}} \\
& +E_{\mathrm{JT}} \sum_{\mathbf{i}}\left[2\left(q_{2 \mathbf{i}} \tau_{x \mathbf{i}}+q_{3 \mathbf{i}} \tau_{z \mathbf{i}}\right)+\left(q_{2 \mathbf{i}}^{2}+q_{3 \mathbf{i}}^{2}\right)\right],
\end{aligned}
$$

with $\tau_{x \mathbf{i}}=d_{a \mathbf{i}}^{\dagger} d_{b \mathbf{i}}+d_{b \mathbf{i}}^{\dagger} d_{a \mathbf{i}}$ and $\tau_{z \mathbf{i}}=d_{a \mathbf{i}}^{\dagger} d_{a \mathbf{i}}-d_{b \mathbf{i}}^{\dagger} d_{b \mathbf{i}}$, where $d_{a \mathbf{i}}$ $\left(d_{b \mathbf{i}}\right)$ is an annihilation operator for an $e_{g}$ electron in the $d_{x^{2}-y^{2}}\left(d_{3 z^{2}-r^{2}}\right)$ orbital at site $\mathbf{i}, \mathbf{a}$ is the vector connecting nearest-neighbor sites, and $t_{\gamma \gamma^{\prime}}^{\mathrm{a}}$ is the hopping amplitude [6] given by $t_{a a}^{\mathbf{x}}=-\sqrt{3} t_{a b}^{\mathbf{x}}=-\sqrt{3} t_{b a}^{\mathbf{x}}=3 t_{b b}^{\mathbf{x}}=3 t / 4$ for $\mathbf{a}=\mathbf{x}$ 
and $t_{a a}^{\mathbf{y}}=\sqrt{3} t_{a b}^{\mathbf{y}}=\sqrt{3} t_{b a}^{\mathbf{y}}=3 t_{b b}^{\mathbf{y}}=3 t / 4$ for $\mathbf{a}=\mathbf{y}$ [7]. The second term with the energy $J^{\prime}$ represents the AFM coupling between nearest-neighbor classical $t_{2 g}$ spins normalized to $\left|\mathbf{S}_{\mathbf{i}}\right|=1$. The third term describes the coupling of an $e_{g}$ electron with the $\left(x^{2}-y^{2}\right)$ - and $\left(3 z^{2}-r^{2}\right)$-type JT modes (dimensionless), given by $q_{2 \mathbf{i}}$ and $q_{3 \mathbf{i}}$, respectively. This term is characterized by the static JT energy $E_{\mathrm{JT}}[8]$.

Intuitively, it can be understood that the competition between kinetic and magnetic energies can produce an SAFM state: The system with $J^{\prime}=0$ is a two-dimensional (2D) ferromagnetic (FM) metal to optimize the kinetic energy of $e_{g}$ electrons, while it becomes a 2D AFM insulator at $J^{\prime} \gtrsim t$ to exploit the magnetic energy of the $t_{2 g}$ spins. For smaller but nonzero values of $J^{\prime}$, there occurs a mixture of FM and AFM states one example of which is the CE-type AFM structure at $x=1 / 2$, schematically shown in Fig. 1(a). In this S-AFM state, a 1D conducting path can be defined by connecting nearest-neighbor sites with parallel $t_{2 g}$ spins. Note, however, that the shape of the optimal path is not obvious. A path with a large stabilization energy is needed to construct a stable 2D structure. Thus, our purpose here is to specify the shapes of (quasi-)stable 1D paths in the S-AFM manifold.

Let us start with the case of $E_{\mathrm{JT}}=0$ and no electron correlation, which allows us to illustrate clearly the importance of topology in the present problem. In Fig. 1(b), the energies per site analytically obtained for various states are plotted as a function of $J^{\prime}$ : In a 2D FM metal, the kinetic-energy gain is reduced by $2 J^{\prime}$, due to the loss of magnetic energy per site. In a $2 \mathrm{D}$ AFM insulator, the magnetic-energy gain per site is $2 J^{\prime}$. In S-AFM states, the optimized periodicity $M$ for a $1 \mathrm{D}$ path along the $a$-axis direction is numerically found to be given by $M=2 / n$, in agreement with Ref. [9], where $n(=1-x)$ is the $e_{g}$-electron number per site. Thus, $M$ is set as 4 at $x=1 / 2$, leading to $2^{4}$ paths, categorized into the four types shown in Fig. 1(b). The energy corresponding to each path is calculated and it is found that the S-AFM state with zigzag3 path is stabilized for $0.1 t \lesssim J^{\prime} \lesssim 0.35 t$. In the rest of the paper, this energy diagrams will not be shown, but a "window" for a stabilized S-AFM state always exists for $J^{\prime}$ of the order of $0.1 t$ at other densities and for $E_{\mathrm{JT}} \neq 0$. As shown in Fig. 1(c), the straight and zigzag1 paths lead to a metal, while the zigzag2 and zigzag3 paths induce a band-insulator. Since it has a larger bandgap (equal to $t$ ), the zigzag3 path is stabilized at $x=1 / 2$.

The S-AFM structure with zigzag3 path is nothing but the well-known CE-type AFM state, and our analysis predicts that this state is very stable at $x=1 / 2$. However, the same analysis for $x \geq 2 / 3$ does not lead to a zigzag path as the optimized one but a straight one, which disagrees with experiment. Thus, it is necessary to find a quantity other than the energy to discuss the possible preferred paths that may arise from a full calculation including nonzero $E_{\mathrm{JT}}$ and Coulomb interactions. Re- considering the results at $x=1 / 2$ lead us to the idea that the number of vertices along the path, $N_{\mathrm{v}}$, may provide the key difference among paths. A confirmation of this idea is given by the calculation of energies for the $2^{6}$ and $2^{8}$ paths at $x=2 / 3(M=6)$ and $3 / 4(M=8)$, respectively. As shown in Fig. 1(d), the energies can be grouped in $(M / 2+1)$-fold bands, each of which is characterized by $N_{\mathrm{v}}(=0,2, \cdots, M)$. This suggests that $N_{\mathrm{v}}$, a topological feature, is relevant for the physics of the S-AFM states.
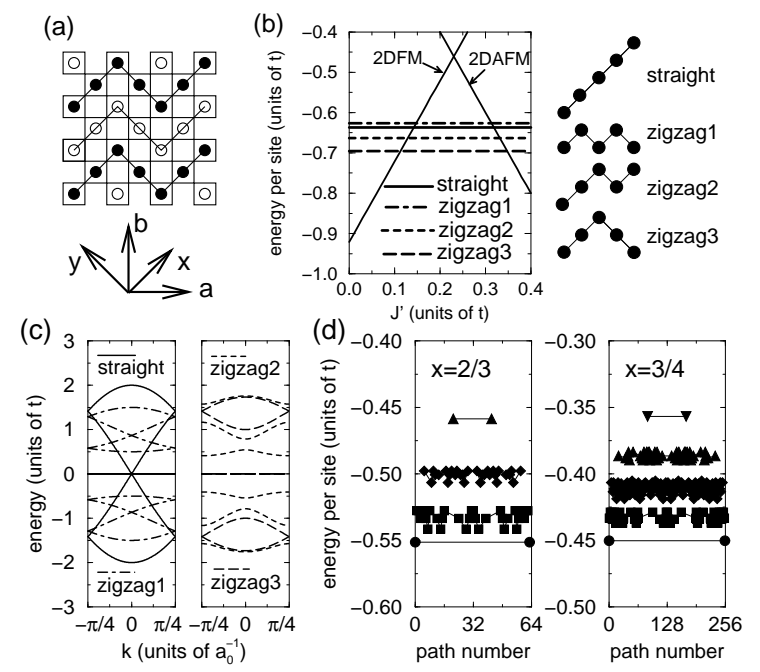

FIG. 1. (a) Schematic view of the S-AFM structure in the $a-b$ plane with oxygens at the corners of squares. Solid and open circles represent, respectively, up and down $t_{2 g}$ spins. Solid lines indicate the hopping paths for $e_{g}$ electrons. (b) Energy per site vs. $J^{\prime}$ for several magnetic arrangements at $x=1 / 2$ and $E_{\mathrm{JT}}=0$. Four types of paths in the S-AFM state are shown. (c) Dispersion curves for the S-AFM states with straight, zigzag1, zigzag2, and zigzag3 path. Here $a_{0}$ is the lattice constant in the $a-b$ plane. (d) Energies per site for all possible paths at $x=2 / 3$ and $3 / 4$ without JT distortions. Circles, squares, diamonds, up-triangles, and down-triangles denote the results for $N_{\mathrm{v}}=0,2,4,6$, and 8 , respectively.

Let us include the JT distortion to substantiate our ideas. By writing the JT modes in polar coordinates as $q_{2 \mathbf{i}}=q_{\mathbf{i}} \sin \theta_{\mathbf{i}}$ and $q_{3 \mathbf{i}}=q_{\mathbf{i}} \cos \theta_{\mathbf{i}}$, "phase-dressed operators", $\tilde{d}_{a \mathbf{i}}$ and $\tilde{d}_{b \mathbf{i}}$, are introduced as $\tilde{d}_{a \mathbf{i}}=e^{i \theta_{\mathbf{i}} / 2}\left[d_{a \mathbf{i}} \cos \left(\theta_{\mathbf{i}} / 2\right)+\right.$ $\left.d_{b \mathbf{i}} \sin \left(\theta_{\mathbf{i}} / 2\right)\right]$ and $\tilde{d}_{b \mathbf{i}}=e^{i \theta_{\mathbf{i}} / 2}\left[-d_{a \mathbf{i}} \sin \left(\theta_{\mathbf{i}} / 2\right)+d_{b \mathbf{i}} \cos \left(\theta_{\mathbf{i}} / 2\right)\right]$ with $e^{i \theta_{\mathbf{i}} / 2}$ representing the molecular Aharonov-Bohm effect. The amplitude $q_{\mathrm{i}}$ is determined by a meanfield approximation [10], while the phases, $\theta_{\mathbf{i}}$ 's, are interrelated through the Berry-phase connection to provide the winding number $w$ along the $1 \mathrm{D}$ path as $w=\oint_{C} d \mathbf{r} \cdot \nabla \theta /(2 \pi)$ [11, where $C$ forms a closed loop for the periodic-lattice boundary conditions 12 .

Mathematically $w$, a Chern number, is proven to be an integer [10]. In this system, it may be decomposed into two terms as $w=w_{\mathrm{g}}+w_{\mathrm{t}}$. The former, $w_{\mathrm{g}}$, is the geometric term, which becomes 0 (1) corresponding to the periodic (anti-periodic) boundary condition in the $e_{g}$-electron wavefunction. The discussion on the kinetic 
energy leads us to conclude that the state with $w_{\mathrm{g}}=0$ has lower energy than that with $w_{\mathrm{g}}=1$ for $x \geq 1 / 2$ [13], in agreement with the two-site analysis [14. Thus, $w_{\mathrm{g}}$ is taken as zero hereafter.

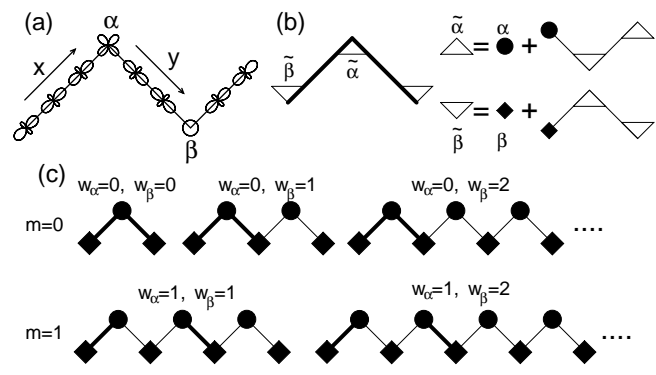

FIG. 2. (a) A typical building block for a 1D path for an $e_{g}$ electron with JT distortions. (b) General structure of the lowest-energy-state path and the renormalization scheme for the vertices $\alpha$ and $\beta$. The thick (thin) line denotes the straight-line part with (without) an $e_{g}$ electron localized on it. The solid circle and diamond denote, respectively, the bare vertices, $\alpha$ and $\beta$, while open up- and down-triangles indicate the renormalized vertices, $\tilde{\alpha}$ and $\tilde{\beta}$. Note that the periodicity of the $1 \mathrm{D}$ path is given by $M=2 / n=2 /(1-x)$. (c) Groups of $1 \mathrm{D}$ paths derived from mother states with $m=0$ and 1. Paths in the first column corresponding to the mother WC structures with $w=2 m+1$, which produce daughter states with $w=2 m+2,2 m+3, \cdots$.

To show that only $N_{\mathrm{v}}$ determines the topological term $w_{\mathrm{t}}$, let us consider the transfer of a single $e_{g}$ electron along the path shown in Fig. 2(a). On the straight-line part in the $x$ - $\left(y\right.$-) direction, the phase is fixed at $\theta_{\mathbf{x}}=2 \pi / 3$ $\left(\theta_{\mathbf{y}}=4 \pi / 3\right)$, because the $e_{g}$-electron orbital is polarized along the transfer direction. This effect may be called an "orbital double-exchange (DE)" in the sense that the orbitals align along the axis direction to make the transfer of the electron smooth, similarly as the FM alignment of $t_{2 g}$ spins in the usual DE mechanism. Thus, $w_{\mathrm{t}}$ does not change on the straight-line part of the path. However, when the electron passes the vertex $\alpha(\beta)$, the phase changes from $\theta_{\mathbf{x}}$ to $\theta_{\mathbf{y}}\left(\theta_{\mathbf{y}}\right.$ to $\left.\theta_{\mathbf{x}}\right)$, indicating that the electron picks up a phase change of $2 \pi / 3(4 \pi / 3)$. Since these two vertices appear in pairs, $w_{\mathrm{t}}(=w)$ is evaluated as $w_{\mathrm{t}}=\left(N_{\mathrm{v}} / 2\right)(2 \pi / 3+4 \pi / 3) /(2 \pi)=N_{\mathrm{v}} / 2$. The phases at the vertices are assigned as an average of the phases sandwiching those vertices, $\theta_{\alpha}=\pi$ and $\theta_{\beta}=0$, to keep $w_{\mathrm{g}}$ invariant. Then, the phases are determined at all the sites once $\theta_{\mathbf{x}}, \theta_{\mathbf{y}}, \theta_{\alpha}$, and $\theta_{\beta}$ are known.

Now we include the cooperative JT effect, important ingredient to determine $\mathrm{COO}$ patterns in the actual manganites. Although its microscopic treatment is involved, we can treat it phenomenologically as a constraint for macroscopic distortions [10], energetically penalizing $w=0$ and $M / 2$ paths. In fact, it is numerically found that $w=1,2, \cdots, M / 2-1$ paths constitute the lowest-energy band and they can be regarded as degenerate, since its bandwidth is about $0.01 t$, much smaller than the inter- band energy difference $(\approx 0.1 t)$. Summarizing, the cooperative JT effect gives us two rules for the localization of $e_{g}$ electrons; (i) they never localize at vertices; (ii) if an electron localizes at a certain site on one of the straight segments in the $x$-direction, the other localizes on one of the straight segments in the $y$-direction.

Applying these rules, we obtain a general structure for the lowest-energy path as shown in Fig. 2(b). Important features are the "renormalized vertices", $\tilde{\alpha}$ and $\tilde{\beta}$, abbreviated notations to represent the set of straight-line parts that do not have $e_{g}$ electrons. The winding number assigned to $\tilde{\alpha}(\tilde{\beta})$ is $1 / 3+w_{\alpha}\left(2 / 3+w_{\beta}\right)$, where the number of vertices included in $\tilde{\alpha}(\tilde{\beta})$ is $1+2 w_{\alpha}\left(1+2 w_{\beta}\right)$. Thus, the lowest-energy path is labeled by the non-negative integers $w_{\alpha}$ and $w_{\beta}$, leading to a total winding number $w=1+w_{\alpha}+w_{\beta}$. Although the topological argument does not determine the precise position at which an $e_{g}$ electron localizes in space, it is enough to regard a charged straight-line part as a "quasi-charge". Since the quasicharges align at equal distance in the WC structure, the corresponding path is labeled by $w_{\alpha}=w_{\beta}=m$, with $m$ a non-negative integer. By increasing $w_{\beta}$ keeping $w_{\alpha}$ fixed, we can produce any non-WC-structure paths with $w_{\alpha}=m$ and $w_{\beta}=m+1, m+2, \cdots$ (see Fig. 2(c)). In this way, the WC structure with $w=2 m+1$ can be considered the "mother state" for all non-WC-structure paths with $w=2 m+2,2 m+3, \cdots$, referred to as the "daughter states". The states belonging to different $m$ 's are labeled by the same $w$, but a large energy barrier exists for the conversion among them, since an $e_{g}$ electron must be moved through a vertex in such a process. Thus, the state characterized by $w$ in the group with $m$, once formed, it cannot decay, even if it is not the lowest-energy state.

Note that the topological argument works irrespective of the detail of $H$, since $w$ is a conserved quantity. However, it cannot single out the true ground state, since the quantitative discussion on the ground-state energy depends on the choice of $H$ and approximations employed. In fact, either the BS or WC structure can be the ground state, but in view of the small energy difference, their relative energy will likely change whenever a new ingredient is added to $H$. Especially, the Coulomb interactions will be important to decide the winner in the competition between these structures 15.

Now we analyze the charge and orbital arrangement in $\mathrm{La}_{1-x} \mathrm{Ca}_{x} \mathrm{MnO}_{3}$, in which the experimental appearance of the BS structure provides key information to specify the $1 \mathrm{D}$ path. Since the quasi-charges exist in a contiguous way in the BS structure, its path is produced from the mother state with $m=0$ (see Fig. 2(c)). In particular, the COO pattern in the shortest $1 \mathrm{D}$ path is uniquely determined as shown in Figs. 3(a)-(c). (To depict these figures, we performed a mean-field calculation for $E_{\mathrm{JT}}=2 t$, but the essential physics does not depend on either the approximation or the choice of $E_{\mathrm{JT}}$.) At $x=1 / 2$, the path 
is characterized by $w=1$ which is the basic mother state with $m=0$. The COO pattern shown in Fig. 3(a) leads to the CE-type AFM state [1]. Those in the paths with $w=2$ and 3 are nothing but the BS structures experimentally observed at $x=2 / 3$ and $3 / 4$ [5]. Note that the short-period zigzag part explains the peculiar feature exhibiting small oscillations in $q_{\mathbf{i}}$ at less- (non-) distorted $\mathrm{Mn}^{4+}$ sites, as suggested in experiments [5].

It may be assumed that the long-range Coulomb interaction $V$ destabilizes the BS structure and transforms it to the WC structure [16], but this is not the case; for the $\mathrm{BS} \rightarrow \mathrm{WC}$ conversion with the help of $V$, an $e_{g}$ electron must be on the vertex in the path with $w=2$ or 3 (see Figs. 3(b) and (c)). This is against rule (i) and thus, the BS structure, once formed, is stable due to the topological condition, even including a weak repulsion $V$.

In the group of $m=0$, the WC structure appears only in the path with $w=1$. Thus, the WC-structure paths with $w=1$ at $x=2 / 3$ and $3 / 4$ are obtained by simple extension of the straight-line part in the path at $x=1 / 2$ (see Figs. 3(d) and (e)). The detailed charge distribution inside the quasi-charge segment is determined by a self-consistent calculation with the JT effect, leading to the WC structure. (A similar result can also be obtained for a weak $V$.) Even if the non-WC structure occurs for $w=1$, it is unstable in the sense that it is easily converted to the WC structure, because no energy barrier exists for an $e_{g}$ electron shift along the straight-line part.

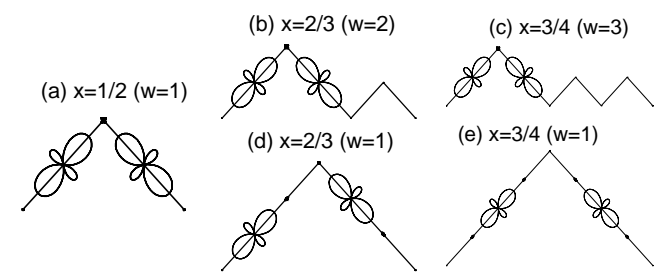

FIG. 3. (a) Path with $w=1$ at $x=1 / 2$ for $E_{\mathrm{JT}}=2 t$. At each site, the orbital shape is shown with its size in proportion to the orbital density. (b) The BS-structure path with $w=2$ at $x=2 / 3$. (c) The BS-structure path with $w=3$ at $x=3 / 4$. (d) The WC-structure path with $w=1$ at $x=2 / 3$. (e) The WC-structure path with $w=1$ at $x=3 / 4$.

The above topological analysis show that (1) the WC structure is made of $w_{\mathrm{WC}}=1$ zigzag paths and that (2) the BS one contains a shorter-period zigzag path with $w_{\mathrm{BS}}=M / 2-1=x /(1-x)$. Note that on the BS path, the less-distorted $\mathrm{Mn}^{4+}$ sites occupy all the vertices $\left(N_{\mathrm{v}}\right.$ equals the number of $\mathrm{Mn}^{4+}$ ions), while the heavily distorted $\mathrm{Mn}^{3+}$ sites appear in pairs (the number of $\mathrm{Mn}^{3+}$ ions equal to 2). Thus, $w_{\mathrm{BS}}$ is rewritten as

$$
w_{\mathrm{BS}}=\frac{N_{\mathrm{v}}}{2}=\frac{\text { Number of } \mathrm{Mn}^{4+} \text { ions }}{\text { Number of } \mathrm{Mn}^{3+} \text { ions }}=\frac{x}{1-x} .
$$

Since $w_{\mathrm{BS}}$ is an integer, we can predict that at specific values of $x\left[=w_{\mathrm{BS}} /\left(1+w_{\mathrm{BS}}\right)\right]$, such as $1 / 2,2 / 3,3 / 4$, etc., nontrivial charge and orbital arrangement will be stabilized in agreement with the experimental observation [5].

Two comments are in order. (1) To understand the observed BS structure, we focussed on paths produced from the $m=0$ mother state, but we could have used a $m \neq 0$ mother state. Thus, we anticipate the existence of a relation similar to Eq. (2) in a higher hierarchy (with much longer periodicity), leading to a devil's staircase structure in the $1 \mathrm{D}$ path. (2) The S-AFM structure with the zigzag path for $w=1$ explains the AFM phase observed in experiment at $x=2 / 3$, but we further predict a similar S-AFM state at $x=3 / 4$ in the WC structure. Our theory also predicts a peculiar spin pattern in the BS structure. Observation of such structures will provide a stringent test for the validity of our topological scenario.

In summary, the BS and WC structures in the manganites have been classified using the winding number $w$ associated with the Berry-phase connection along the zigzag 1D path. Predictions are made for novel spin and orbital states in heavily doped manganites.

T.H. thanks S. Yunoki and C. H. Chen for discussions. T.H. and Y.T. are supported from the Ministry of Education, Science, Sports, and Culture of Japan. E.D. is supported by grant NSF-DMR-9814350.

[1] S.-W. Cheong and C. H. Chen, in Colossal Magnetoresistance, Charge Ordering, and Related Properties of Manganese Oxides, eds. C. N. R. Rao and B. Raveau, (World Scientific, Singapore, 1998).

[2] E. O. Wollan and W. C. Koehler, Phys. Rev. 100, 545 (1955); J. B. Goodenough, ibid., 564 (1955).

[3] P. G. Radaelli et al., Phys. Rev. B59, 14440 (1999); M. T. Fernández-Díaz et al., Phys. Rev. B59, 1277 (1999).

[4] Y. Murakami et al., Phys. Rev. Lett. 80, 1932 (1998).

[5] S. Mori et al., Nature 392, 473 (1998).

[6] In general, $t_{\gamma \gamma^{\prime}}^{\mathbf{a}}$ depends on the relative angle between nearest-neighbor $t_{2 g}$ spins, but for simplicity, only FM or AFM configurations are considered in this work and $t_{\gamma \gamma^{\prime}}^{\mathrm{a}}$ is non-zero only for the FM configuration.

[7] Here $t$ is defined as the hopping amplitude between nearest-neighbor $d_{3 z^{2}-r^{2}}$ orbitals along $z$-axis.

[8] Note that the JT distortions are treated adiabatically.

[9] H. Koizumi et al., Phys. Rev. Lett. 80, 4518 (1998).

[10] T. Hotta et al., Int. J. Mod. Phys. B12, 3437 (1998).

[11] H. Koizumi et al., Phys. Rev. Lett. 81, 3803 (1998).

[12] Note that the concept of winding number $w$, which implicitly assumes mobile electrons, is relevant even for an insulator, provided that it is a band insulator, because the system is described by the superposition of extended wavefunctions with which we can associate $w$.

[13] At $x=0$, the ground state possesses $w_{\mathrm{g}}=1$ to gain the JT energy with an alternate orbital pattern [9].

[14] Y. Takada et al., cond-mat/9906128. 
[15] Qualitatively, the present result will not be altered by the on-site Coulomb repulsion, since the double occupancy of electrons at a site is already suppressed by the JT stabilization mechanism.

[16] Recently, it has been argued that $V$ plays an essential role for the charge-ordered stripe structure forma- tion [T. Mutou and H. Kontani, Phys. Rev. Lett . 83, 3685 (1999)]. However, we believe that its effect should be limited, since if it is too strong, it produces a threedimensional WC. Such a phase is not observed experimentally, instead a simple stacking along $c$-axis is realized. For details, see S. Yunoki et al., cond-mat/9909254. 